\title{
Effets de la vitesse de croissance au pâturage chez des taurillons finis en stabulation: II. Qualité de la viande et composition de la graisse
}

\author{
JL Hornick, A Clinquart, S Gauthier, C Van Eenaeme, L Istasse \\ Service de Nutrition, Fac Méd Vét, Univ de Liège, Sart-Tilman, 43-4000 Liège, Belgique
}

La finition en étable, chez des taurillons qui ont pâturé, est souvent d'autant plus rapide que les performances à l'herbe ont été faibles. La qualité de la viande peut ainsi être modifiée par le biais de la mise à l'herbe mais aussi par le développement de la croissance compensatrice.

Vingt taurillons culards de race Bleu Blanc belge, pesant $250 \mathrm{~kg}$ ont été répartis en trois groupes. Huit animaux ont pâturé une prairie avec une grande disponibilité d'herbe (groupe croissance classique en prairie, CCP). Huit animaux ont pâturé une prairie dont la disponibilité en herbe était réduite (groupe croissance faible en prairie, CFP). Quatre animaux ont été engraissés à l'étable sans passage préalable en prairie (groupe témoin, T). A l'abattage, un segment tricostal a été prélevé. La qualité de la viande et la composition de la graisse ont été déterminées respectivement sur le muscle Longissimus Thoracis et sur des prélèvements de graisse sous-cutanée, intermusculaire et intramusculaire.

Le gain moyen quotidien en prairie a été de $0,54 \mathrm{~kg} / \mathrm{j}$ pour le groupe CFP et de $1,08 \mathrm{~kg} / \mathrm{j}$ pour le groupe CCP. Les performances en étable ont été de 1,$54 ; 1,26$ et $1,29 \mathrm{~kg} / \mathrm{j}$ pour les groupes CFP, CCP et $\mathrm{T}$ respectivement $(P>0,1)$. Le $\mathrm{pH}$ post-mortem de la viande a chuté plus rapidement chez les animaux ayant pâturé $(P<0,01)$ mais les différences se sont estompées $48 \mathrm{~h}$ après l'abattage. La viande a été plus sombre deux jours après l'abattage mais aucune différence significative n'a été trouvée au niveau des paramètres de couleurs. Les pertes de jus et les pertes d'eau à la cuisson mesurées 9 jours après l'abattage ont été semblables entre les groupes. La force de cisaillement a été significativement plus faible dans les groupes ayant pâturé $(P<0,05)$. L'analyse de la composition chimique du muscle a révélé une plus grande proportion de cendres et de protéines et une plus faible proportion d'extrait éthéré $(P<0,001)$ chez les animaux mis à l'herbe. La graisse de ces animaux contenait une proportion plus faible de $\mathrm{C} 14: 0, \mathrm{C} 16: 0$ et d'acides gras saturés $(P<0,01 ; P<0,001 ; P<0,05)$ et généralement une proportion plus élevée d'acides gras à chaînes plus longues et insaturés (C18:0, C18:1, C18:2 et C18:3). Les concentrations de cholestérol dans la viande ont été plus élevées dans les groupes ayant pâturé $(P>0,2$ et $P<0,05$ dans les groupes $C F P$ et $C C P$ respectivement).

En conclusion, la vitesse de croissance au pâturage n'a pas influencé la qualité de la viande ni la composition de la graisse. Comparés à des animaux engraissés en stabulation, les taurillons mis à l'herbe ont produit une viande plus tendre et plus maigre et une graisse contenant plus de chaînes longues et insaturées.

\begin{tabular}{|c|c|c|c|}
\hline$T$ & $\mathrm{CCP}$ & CFP & SED \\
\hline $6,15^{a}$ & $6,60^{\mathrm{b}}$ & $6,48^{b}$ & 0,08 \\
\hline $5,92^{\mathrm{a}}$ & $6,20^{b}$ & $6,22^{b}$ & 0,09 \\
\hline $5,63^{a}$ & $5,59 \mathrm{a}$ & $5,55^{a}$ & 0,08 \\
\hline $44,13^{a}$ & $41,15^{\circ}$ & $42,63^{a b}$ & 1,67 \\
\hline $0,98^{\mathrm{a}}$ & $1,00^{a}$ & $0,98^{a}$ & 0,05 \\
\hline $5,31^{a}$ & $4,88^{a}$ & $4,50^{\mathrm{a}}$ & 0,77 \\
\hline $21,68^{a}$ & $22,60^{\mathrm{a}}$ & $21,94^{a}$ & 1,48 \\
\hline $44,15^{a}$ & $29,42^{b}$ & $31,25^{b}$ & 5,29 \\
\hline $4,19^{a}$ & $5,60^{\mathrm{b}}$ & $6,11^{b}$ & 0,44 \\
\hline $84,13^{a}$ & $91,07^{b}$ & $89,22^{b}$ & 1,12 \\
\hline $6,25^{a}$ & $3,76^{\mathrm{b}}$ & $3,16^{b}$ & 0,97 \\
\hline $1,93^{a}$ & $2,86^{b}$ & $2,59^{a b}$ & 0,44 \\
\hline $52,99 a$ & $50,8^{b}$ & $50,80^{b}$ & 1,03 \\
\hline
\end{tabular}

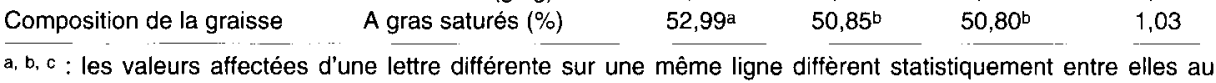
seuil $P<0,1 ; P<0,01$ ou $P<0,001$. Remerciements: Travail réalisé avec la collaboration financière de I'IRSIA (Institut pour l'Encouragement de Recherche dans l'Industrie et l'Agriculture, Bruxelles, Belgique). 